\title{
A breakthrough in the functional evaluation of the inspiratory muscle pump
}

\author{
T.L. Clanton
}

In this issue, HART et al. [1] describe a new respiratory muscle endurance test that could greatly impact the understanding of clinical respiratory muscle biology in a variety of patient groups. In a post-genomic era, in which breakthroughs in both the understanding of diseases at a molecular level and the treatments based on designer molecules occur daily, why should this warrant news?

Look to the cardiologists. Cardiologists do not hesitate to consider developing aggressive treatment options (as ambitious as gene therapy or stem cell implantation) to combat the influence of hypertension or cardiac failure. These approaches are considered even though ventricular muscle disease does not represent the underlying cause of the disorder. Pulmonologists have traditionally been much more conservative. Their research and treatment modalities have focused exclusively on the underlying pathology of the lung, even though the predominant lifechanging symptom of the disease is dyspnoea, which is largely believed to be secondary to increased respiratory muscle activity. This strategy has been appropriate both in an environment in which the understanding of the effects of lung disease on the chest wall is limited and when the available testing procedures and treatment options are poorly developed. In contrast, cardiologists develop new ideas for treatment based on an ongoing database of information on left ventricular dysfunction that has been growing for decades. They have invested in technology to quantify and track ventricular wall dysfunction, metabolic abnormalities and contractile deficits. In most institutions, pulmonologists have little or no capacity to track the extent of chest wall disorders in lung disease, as the appropriate tests and procedures have not been adequately developed. The most sophisticated tests available in many clinical settings are maximum inspiratory and expiratory pressures, which are inadequate when viewed in isolation, as illustrated by HART et al. [1].

Three major contributions to the understanding of clinical respiratory muscle biology are provided in this study. First, the authors have redefined the

Pulmonary and Critical Care Medicine, Ohio State University, Dorothy M. Dans Heart and Lung Research Institute, OH, USA.

Correspondence: T.L. Clanton, Pulmonary and Critical Care Medicine, Ohio State University, Dorothy M. Dans Heart and Lung Research Institute, OH, USA. Fax: 1 6142934799. E-mail: clanton.1@ osu.edu practical use of a popular pressure-time index concept that describes the load seen by the respiratory chest wall muscles $[2,3]$. The ability to quantify this parameter in a practical way with respiratory loading has been a major stumbling block in evaluating respiratory muscle and chest wall function. Secondly, they have described a new respiratory muscle endurance testing procedure that overcomes several of the limitations of previously described techniques. Most notably, it quantifies a measure of respiratory "work capacity" that is corrected for the individual's spontaneous breathing pattern, making it unnecessary to rigidly control breathing rhythm, a problem that has added to uncertainties about these measurements when using other tests. In addition, its outcome parameter should more clearly differentiate the "endurance" properties from the "strength" properties of the muscles, through measurement of the breathing pattern-corrected pressure-time index (PTPoes/ $P$ oes,max). Other popular and useful techniques developed in recent years (e.g. the incremental test of MARTYN et al. [4]), have theoretical difficulties with respect to separating these two basic properties of muscle. Thirdly, the investigators have demonstrated that patients with a form of interstitial lung disease have an underlying disorder that translates into a reduction in the working capacity (endurance) of the respiratory muscles. These patients exhibit completely normal inspiratory muscle strength.

The work of HART et al. [1] adds to the armamentarium through which pulmonary scientists can begin building a database to understand the nature and time course of chest wall disorders in lung disease. Coupled with emerging technologies such as noninvasive measures of diaphragm twitch strength, measures of respiratory muscle thickness and confirmation, analysis of the energetic costs of respiratory muscle activity in exercise, and morphological measurements of respiratory muscles from biopsies, a picture is developing that suggests that each respiratory disease may have its own unique complement of chest wall and respiratory muscle adaptations. Do these reflect disorders? Possibly not, but with more data provided by such tests, new treatment paradigms may be designed to further promote selective and positive muscle and chest wall adaptations in patients with chronic lung disease, providing new weapons against the smothering influence dyspnoea has on their lives. 


\section{References}

1. Hart N, Hawkins $\mathrm{P}$, Hamnegård $\mathrm{C}-\mathrm{H}$, Green $\mathrm{M}$, Moxham J, Polkey MI. A novel clinical test of respiratory muscle endurance. Eur Respir J 2002; 19: 232-239.

2. Bellemare F, Grassino A. Effect of pressure and timing of contraction on human diaphragm fatigue. J Appl Physiol 1982; 53: 1190-1195.
3. Zoccchi L, Fitting JW, Majani U, et al. Effect of pressure and timing of contraction on human rib cage muscle fatigue. Am Rev Respir Dis 1993; 147: 857864.

4. Martyn JB, Moreno RH, Pare PD, Pardy RL. Measurement of inspiratory muscle performance with incremental threshold loading. Am Rev Respir Dis 1987; 135: 919-923. 